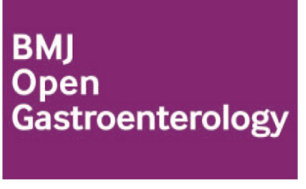

To cite: Prince D, Popuri R, Majumdar A. An essential accessory. BMJ Open Gastro 2021;0:e000770. doi:10.1136/ bmjgast-2021-000770

Received 16 August 2021 Accepted 7 September 2021

\title{
An essential accessory
}

\author{
David Prince (D , ${ }^{1}$ Radha Popuri, ${ }^{2}$ Avik Majumdar (D) ${ }^{1,3}$
}

\begin{abstract}
A young adult male was referred for a second opinion of deranged liver biochemistry. He initially presented two years prior with abdominal pain, lethargy and fevers due to a segment two pyogenic liver abscess. He received empirical antibiotic therapy to resolution. Computed tomography for abscess follow-up revealed an intrahepatic inferior vena cava thrombus. He was anti-coagulated with warfarin. He was lupus anticoagulant positive and had a highly positive beta-2 glycoprotein antibody on serial measurement and was diagnosed with anti-phospholipid syndrome. On current review, the patient had no clinical stigmata of chronic liver disease. There were dilated veins on the supraumbilical abdominal and chest walls. There was mild hepatomegaly but no splenomegaly. Laboratory investigations revealed mildly cholestatic liver function tests with hyperbilirubinaemia $(40 \mu \mathrm{mol} / \mathrm{L})$ but no liver synthetic dysfunction. Serological screening did not reveal any cause of chronic liver disease. The patient underwent multiphase abdominal CT and formal hepatic venography. What is the diagnosis and describe the hepatic venous outflow?
\end{abstract}

\section{QUESTION}

A young adult male was referred for a second opinion of deranged liver biochemistry. He

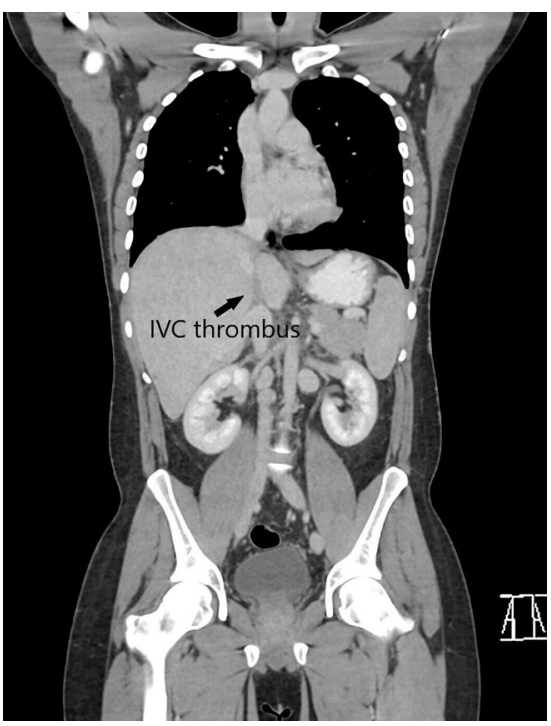

Figure 1 Venous phase CT demonstrating thrombus occluding the entire intrahepatic IVC. The hepatic veins are not visualised however a dilated accessory inferior right hepatic vein (AIRHV) can be seen. IVC, inferior vena cava.



Figure 2 Cavogram of patient IVC superior to thrombosis. The hepatic veins could not be catheterised. IVC, inferior vena cava.

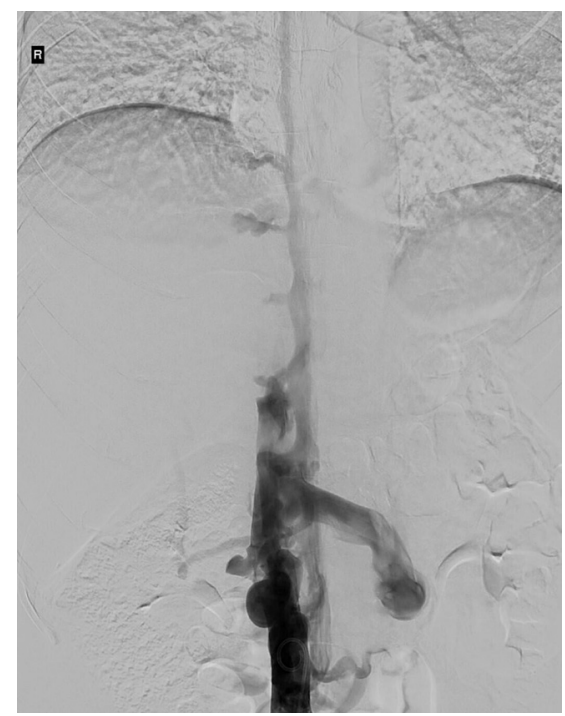

Figure 3 Cavogram of the patent IVC inferior to the thrombosis.

initially presented 2 years prior with abdominal pain, lethargy and fevers due to a segment two pyogenic liver abscess. He received empirical antibiotic therapy to resolution. CT for abscess follow-up revealed an intrahepatic inferior vena cava (IVC) thrombus. He was anticoagulated with warfarin. He was lupus anticoagulant positive and had a highly positive beta- 2 glycoprotein antibody on serial 




Figure 4 Selective catheterisation of the AIRHV with angiography demonstrating near complete occlusion of the right and middle hepatic vein ostia with aberrant intrahepatic venous collaterals allowing hepatic venous outflow via the AlHRV. Blue arrows denote direction of blood flow.

measurement and was diagnosed with antiphospholipid syndrome (APS).

On current review, the patient had no peripheral clinical stigmata of chronic liver disease. There were dilated veins on the supraumbilical abdominal and chest walls. There was mild hepatomegaly but no splenomegaly. Laboratory investigations revealed mildly cholestatic liver function tests with hyperbilirubinaemia $(40 \mu \mathrm{mol} / \mathrm{L})$ but no liver synthetic dysfunction. Serological screening did not reveal any cause of chronic liver disease. He did not have antibodies to Echinoccoccus granulosus or Fasciola hepatica.

The patient underwent multiphase abdominal CT and formal hepatic venography (figures 1-6).

What is the diagnosis and describe the hepatic venous outflow?

\section{ANSWER}

There is ongoing occlusive thrombus of the intrahepatic IVC and the hepatic vein ostia in the context of APS (figures 1-3). The main hepatic veins are not visualised; however, a dilated accessory inferior right hepatic vein

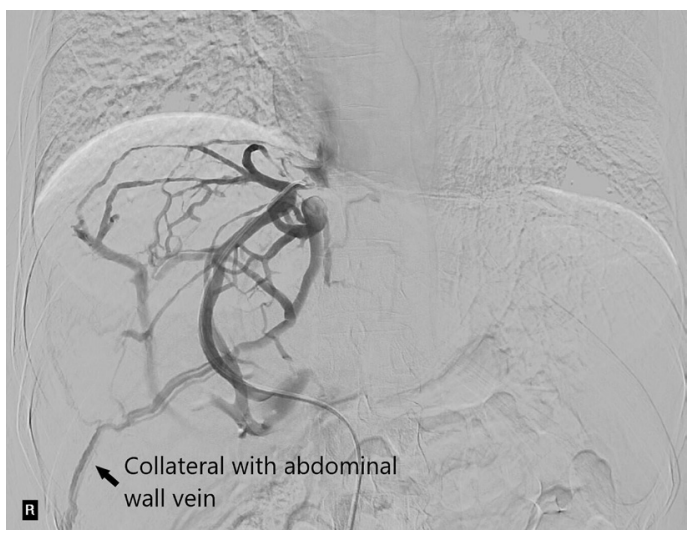

Figure 5 Selective catheterisation of the AIRHV with further demonstration of aberrant intrahepatic and extrahepatic collaterals to allow for hepatic venous outflow.

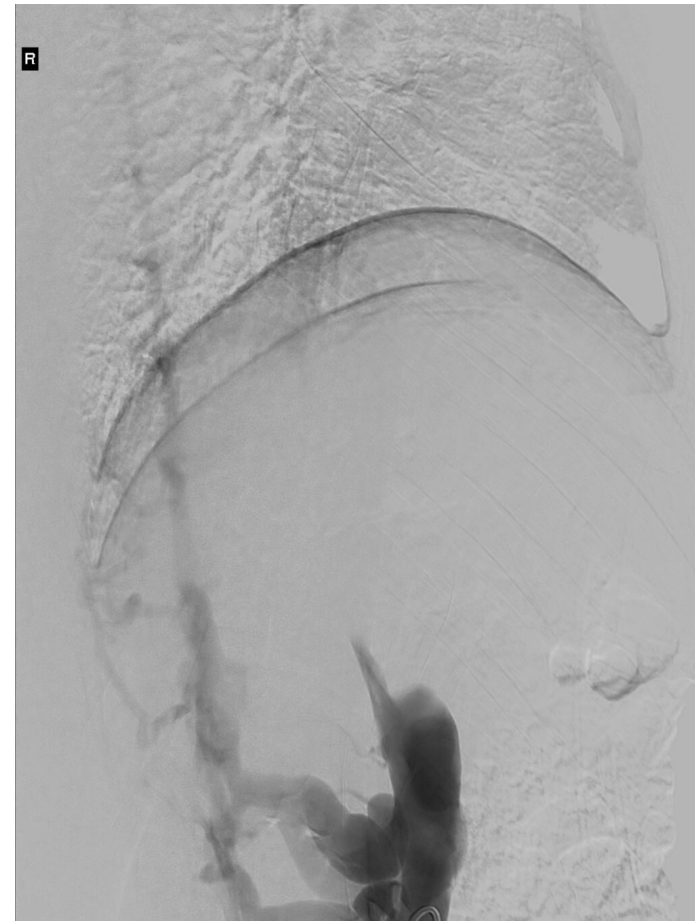

Figure 6 Lateral angiographic view demonstrating occluded IVC with collateralisation of paralumbar veins.

(AIRHV) is seen. Figure 2 is a cavogram of patent IVC superior to the thrombosis. The hepatic veins could not be catheterised.

Unusually in this case, there were no features of BuddChiari syndrome (BCS). BCS is characterised by partial or complete hepatic venous outflow obstruction, which causes increased hepatic sinusoid pressure and hepatocyte hypoxia. ${ }^{1}$ Patients may present acutely with abdominal pain, ascites, hepatomegaly and liver failure, but can occasionally be asymptomatic and present subsequently with features of chronic liver disease. ${ }^{2}$

In this case, hepatic venous outflow was predominantly via a dilated AIRHV, which drained directly into the IVC below the thrombosis (figure 4). Aberrant collaterals from the middle hepatic to the AIRHV were demonstrated on venography (figures 4-5). Blood from the IVC returns to the right atrium via collateralised paralumbar and azygos veins (figure 3, figure 6). Accessory right hepatic veins are present in $30 \%$ of patients ${ }^{3}$ and in this case, the accessory vein was an essential compensatory vessel to allow hepatic venous outflow and avoid BCS.

Contributors All authors contributed equally

Funding The authors have not declared a specific grant for this research from any funding agency in the public, commercial or not-for-profit sectors.

Competing interests None declared.

Patient consent for publication Not applicable.

Provenance and peer review Not commissioned; internally peer reviewed.

Data availability statement Data sharing not applicable as no datasets generated and/or analysed for this study.

Open access This is an open access article distributed in accordance with the Creative Commons Attribution Non Commercial (CC BY-NC 4.0) license, which permits others to distribute, remix, adapt, build upon this work non-commercially, 
and license their derivative works on different terms, provided the original work is properly cited, appropriate credit is given, any changes made indicated, and the use is non-commercial. See: http://creativecommons.org/licenses/by-nc/4.0/.

\section{ORCID iDs}

David Prince http://orcid.org/0000-0001-8087-0548

Avik Majumdar http://orcid.org/0000-0003-2975-4327

\section{REFERENCES}

1 Bansal V, Gupta P, Sinha S, et al. Budd-Chiari syndrome: imaging review. Br J Radiol 2018;91:20180441.

2 Janssen HLA, Garcia-Pagan J-C, Elias E, et al. Budd-Chiari syndrome: a review by an expert panel. $J$ Hepatol 2003;38:364-71.

3 pp.De Cecchis L, Hribernik M, Ravnik D, et al. Anatomical variations in the pattern of the right hepatic veins: possibilities for type classification. J Anat 2000:197 Pt 3:487-93. 